\title{
Inflammatory dysregulation of monocytes in pediatric patients with obsessive- compulsive disorder
}

\author{
Natalia Rodríguez ${ }^{1 \dagger}$, Astrid Morer ${ }^{2,6,7 \dagger}$, E. Azucena González-Navarro 3,6 Carles Serra-Pages ${ }^{3,5,6}$, Daniel Boloc ${ }^{4}$, \\ Teresa Torres ${ }^{1}$, Susana García-Cerro ${ }^{1}$, Sergi Mas ${ }^{1,6,7}$, Patricia Gassó ${ }^{1,6}$ and Luisa Lázaro ${ }^{2,4,6,7^{*}}$ (D)
}

\begin{abstract}
Background: Although the exact etiology of obsessive-compulsive disorder (OCD) is unknown, there is growing evidence of a role for immune dysregulation in the pathophysiology of the disease, especially in the innate immune system including the microglia. To test this hypothesis, we studied inflammatory markers in monocytes from pediatric patients with OCD and from healthy controls.

Methods: We determined the percentages of total monocytes, CD16+ monocytes, and classical (CD14 ${ }^{\text {high }}$ CD16-), intermediate $\left(\mathrm{CD} 14^{\text {high }} \mathrm{CD} 16^{\text {low }}\right)$, and non-classical $\left(\mathrm{CD} 14^{\text {low }} \mathrm{CD} 16^{\text {high }}\right)$ monocyte subsets in 102 patients with early-onset OCD and in 47 healthy controls. Moreover, proinflammatory cytokine production (GM-CSF, IL-1 $\beta$, IL-6, IL-8, and TNF-a) was measured by multiplex Luminex analysis in isolated monocyte cultures, in basal conditions, after exposure to lipopolysaccharide (LPS) to stimulate immune response or after exposure to LPS and the immunosuppressant dexamethasone.

Results: OCD patients had significantly higher percentages of total monocytes and CD16+ monocytes than healthy controls, mainly due to an increase in the intermediate subset but also in the non-classical monocytes. Monocytes from OCD patients released higher amounts of GM-CSF, IL-1 $\beta, I L-6, I L-8$, and TNF- $a$ than healthy controls after exposure to LPS. However, there were no significant differences in basal cytokine production or the sensitivity of monocytes to dexamethasone treatment between both groups. Based on monocyte subset distribution and cytokine production after LPS stimulation, patients receiving psychoactive medications seem to have an intermediate inflammatory profile, that is, lower than non-medicated OCD individuals and higher than healthy controls.
\end{abstract}

Conclusions: These results strongly support the involvement of an enhanced proinflammatory innate immune response in the etiopathogenesis of early-onset OCD.

Keywords: Obsessive-compulsive disorder, Children, Immune system, Microglia, Monocytes, Cytokines, Inflammation

\section{Background}

Obsessive-compulsive disorder (OCD) is a neuropsychiatric disease characterized by recurrent obsessions and/ or compulsions that are distressing, time-consuming, or significantly impairing [1]. It is the fourth most common psychiatric illness, with a lifetime prevalence of $1-3 \%$

\footnotetext{
* Correspondence: LLAZARO@clinic.cat

${ }^{\dagger}$ Equal contributors

${ }^{2}$ Department of Child and Adolescent Psychiatry and Psychology, Institute of Neurosciences, Hospital Clinic de Barcelona, Barcelona, Spain

${ }^{4}$ Department of Medicine, University of Barcelona, Barcelona, Spain

Full list of author information is available at the end of the article
}

[2]. OCD presents a bimodal distribution for age at onset with a peak at 12-14 years (early-onset) and another at 20-22 years (late-onset). Indeed, in 30-50\% of patients, obsessive-compulsive symptoms start to develop in childhood [3, 4]. It has been proposed that childhood-onset OCD may be a distinct form of the disorder with different etiopathogenic mechanisms $[4,5]$.

Evidence from different lines of research suggest a possible role of immune dysregulation in the pathophysiology of OCD [6-8]. The hypothesis of immune dysregulation in OCD was originally based on the association found between streptococcal infections and the abrupt 
onset of obsessive-compulsive symptoms. In this regard, Swedo et al. reported an increased frequency of obsessivecompulsive symptoms and OCD in children with Sydenham's chorea (SC), a delayed neurological complication following streptococcal infections [9]. The syndrome was named Pediatric Autoimmune Neuropsychiatric Disorders Associated with Streptococcal Infections (PANDAS), a subset of childhood tic disorder and/or OCD which may be causally related to group $A \beta$-hemolytic streptococcal infection $[10,11]$.These findings were confirmed in subsequent studies $[12,13]$ and were also extended to patients with a past streptococcal infection without SC symptoms [7, 14]. Although there is some controversy, it has been hypothesized that this phenomenon may be due to the production of antibodies against basal ganglia structures $[6,7,11]$. In addition to streptococcus infection, other infectious diseases including Borrelia burgdorferi, mycoplasma, Toxoplasma gondii, or Borna disease virus have been associated with OCD [7, 15]. As different etiologic factors, both infectious and non-infectious, may be involved, the term PANDAS evolved later to a more wide spectrum of acute neuropsychiatric syndrome named PANS (pediatric acute-onset neuropsychiatric syndrome) [15]. These findings have led to increased interest in the involvement of immunological mechanisms in the etiopathogenesis of OCD, regardless of whether it fulfills the diagnostic criteria for PANDAS or not. Some studies have suggested a dysregulation of the immune function in OCD based on alterations in innate and adaptive immune-related parameters such as proinflammatory cytokine levels [16-18], antineural antibodies $[8,19,20]$, or hypothalamus-pituitary-adrenal axis dysregulation $[21,22]$. However, the results to date are inconclusive.

Several lines of evidence suggest that dysfunction of innate immunity, including the microglia, the brain's resident immune cells derived from the monocyte lineage, may occur in a number of neuropsychiatric conditions [23-26]. The innate immune system has a pivotal role in initiating, directing, and prolonging the immune response. The main immune cells involved in innate response are monocytes, which circulate in the blood, promote rapid responses, and orchestrate inflammation through the release of proinflammatory cytokines [27]. In addition, this system participates in neuroprotection and neurodevelopment [28]. Human blood monocytes are a heterogeneous population that can be segregated into three functionally different subsets based on their expression of CD14 and CD16: classical (CD14 $\left.{ }^{\text {high }} \mathrm{CD} 16-\right)$, intermediate $\left(\mathrm{CD} 14^{\text {high }} \mathrm{CD} 16^{\text {low }}\right)$, and non-classical $\left(\mathrm{CD} 14^{\text {low }} \mathrm{CD} 16^{\text {high }}\right)$ monocytes [29]. CD16+ monocytes (including intermediate and non-classical monocytes [30]) and specifically intermediate monocytes have been termed "proinflammatory" based on their higher production of inflammatory cytokines such as interleukin 6 (IL-6), IL-1 $\beta$, and tumor necrosis factor alpha (TNF- $\alpha$ ) and because of the expansion of these cells in infections and inflammatory diseases [30, 31]. However, the particular state of these cells in OCD is not well known.

Hence, to improve our understanding of the potential role of the innate immune dysregulation in early-onset OCD, we aimed (1) to characterize the monocyte subsets in pediatric patients with OCD and healthy controls, (2) to examine the functionality of cultured monocytes by measuring proinflammatory cytokine and chemokine production after exposure to immune regulators in both OCD and controls, and (3) to explore the relationship of these immune parameters with clinical characteristics of OCD patients.

\section{Methods \\ Subjects}

One hundred and two children and adolescents aged between 8 and 19 years with a current diagnosis of obsessive-compulsive disorder (OCD) according to DSMIV [32] criteria were recruited from the Department of Child and Adolescent Psychiatry and Psychology at the Hospital Clínic in Barcelona. The Spanish version [33] of the semi-structured diagnostic interview K-SADS-PL (Schedule for Affective Disorders and Schizophrenia for School-Age Children-Present and Lifetime Version) [34] was administered with both parents and the child as informant in order to establish the diagnosis of OCD and to assess past and current psychiatric comorbidity. Patients with psychiatric comorbidities other than OCD were not excluded. In addition, due to the naturalistic design of the study, we allowed the inclusion of patients receiving psychoactive medications. Exclusion criteria included intellectual disability, neurological disorders, and known inflammatory disease. The age of onset of OCD was defined as the age at which patients first displayed significant distress or impairment associated with obsessivecompulsive symptoms. OCD severity was measured at the time of admission using the Children's Yale-Brown Obsessive-Compulsive Scale (CY-BOCS) [35], whose maximum score is 40 points.

Forty-seven healthy controls (age range 11-18 years) were recruited from schools in the same geographical region. Controls and their parents were interviewed with the Spanish version [33] of the K-SADS-PL to assess current and past psychopathology. Subjects with a personal history of psychiatric disorders, intellectual disability, and other neurological illness or known inflammatory disease were excluded.

All subjects were recruited between 2010 and 2014 . All participants denied the use of alcohol or other recreational drugs during the semi-structured interview. All procedures were approved by the hospital's ethics committee. Written informed consent was obtained from all 
parents and verbal informed consent was given by all subjects following an explanation of the procedures involved.

\section{Isolation of peripheral blood mononuclear cell (PBMC)}

Blood samples of both OCD patients and healthy individuals were collected in BD Vacutainer tubes containing acid citrate dextrose (Becton Dickinson, Franklin Lakes, New Jersey, USA) for immune cell preparation. All samples were taken during the morning, before 12 p.m. Peripheral blood mononuclear cell (PBMC) suspensions were prepared by density gradient centrifugation over Ficoll-Plaque (GE Healthcare Bio-Science AB, Uppsala, Sweden) at $750 \times g$ for $20 \mathrm{~min}$ at $18{ }^{\circ} \mathrm{C}$. After washing the interphase cells with phosphate-buffered saline (PBS), PBMCs were frozen in fetal bovine serum (FBS) (Life Technologies, Carlsbad, CA, USA) containing 10\% dimethyl sulfoxide (Sigma-Aldrich, St. Louis, MO, USA) and stored in liquid nitrogen until subsequent trials in order to test patient and control immune cells in the same series of experiments and thus to avoid batch effects/interassay variation.

For subsequent analysis, cryopreserved PBMCs were rapidly thawed and washed with RPMI 1640 supplemented with $2 \mathrm{mM}$ L-glutamine, 10\% FBS, 100 units $/ \mathrm{mL}$ penicillin, $100 \mu \mathrm{g} / \mathrm{mL}$ streptomycin (Life Technologies, Carlsbad, CA, USA), and 50 units $/ \mathrm{mL}$ Benzonase ${ }^{\circ}$ nuclease (Sigma-Aldrich, Saint Louis, MO, USA). Cells were counted using an Ac.T diff ${ }^{\mathrm{m}}$ automated analyzer (Beckman Coulter, Miami, FL, USA).

\section{Flow cytometric analysis}

To investigate whether the composition of peripheral monocytes is altered in early-onset OCD, a FACS-based analysis was performed. For this purpose, an aliquot of thawed PBMC containing at least 50,000 cells was washed with PBS and stained with fixable viability dye (eBioscience, San Diego, CA, USA) for $10 \mathrm{~min}$ at room temperature, followed by surface staining for $20 \mathrm{~min}$ with anti-HLA-DR-V500, anti-CD14-Pe-Cy7, and antiCD16-FITC antibodies (BD Pharmingen, San Diego, CA, USA). After incubation, cells were analyzed by flow cytometry on a BD FACSCanto II (BD Bioscience, San Jose, CA, USA) using appropriate color compensation to correct for spectral overlap and autofluorescence. Data were analyzed with FlowJo version 10.1r5 software (Ashland, OR, USA). The gating strategy for identification of monocyte subsets is shown in Additional file 1: Figure S1. Firstly, the putative monocyte population was gated based on forward and side scatter profiles. Then, after selecting viable cells, monocytes were discriminated from the rest of leukocytes based on high expression of HLA-DR. Finally, the different patterns of expression of CD14 and CD16 allowed the identification of the three monocyte subsets: classical (CD14 $\left.{ }^{\text {high }} \mathrm{CD} 16-\right)$, intermediate $\left(\mathrm{CD} 14^{\text {high }} \mathrm{CD} 16^{\text {low }}\right)$, and non-classical $\left(\mathrm{CD} 14^{\text {low }} \mathrm{CD} 16\right.$ high) monocytes. Isotype-matched control antibodies were used to determine the cutoff between negative and positive CD16. The combination of intermediate and nonclassical monocytes in a single population was considered as CD16+ monocytes. Total monocytes were considered as the sum of classical, intermediate, and non-classical monocytes. Percentages of total monocytes, classical, intermediate, and non-classical monocyte subsets, and all CD16+ monocytes were assessed. Only samples with at least 200 total monocytes were included in the statistical analysis (91 early-onset OCD and 34 healthy controls).

\section{Purification, culture, and stimulation of peripheral monocytes}

For the subsequent assessment of monocyte functionality, monocytes were purified from PBMC by negative selection using an indirect magnetic labeling system (MACS, Miltenyi Biotec, Auburn, CA, USA) following the manufacturer's instructions. Briefly, non-monocytes, such as T cells, NK cells, B cells, and dendritic cells, were labeled using a cocktail of biotin-conjugated antibodies and anti-biotin microbeads. These magnetically labeled non-monocytes were depleted by being retained on a $\mathrm{MACS}^{\circ}$ Column in the magnetic field of a MACS Separator while the unlabeled monocytes passed through the column. This kit allowed the simultaneous enrichment of classical, intermediate, and non-classical monocytes. Purity of isolated monocytes was checked by flow cytometry (routinely $>95$ ). No monocytes were purified from one sample from an OCD patient and one from a healthy control, despite following the same protocol.

Purified monocytes (101 OCD patients and 46 controls) were seeded at a density of $1.5 \times 10^{5}$ cells/well on 24-well plates and allowed to rest for $2 \mathrm{~h}$ in RPMI 1640 supplemented with $2 \mathrm{mM}$ L-glutamine, 10\% FBS, 100 units $/ \mathrm{mL}$ penicillin, and $100 \mu \mathrm{g} / \mathrm{mL}$ streptomycin (Life Technologies, Carlsbad, CA, USA) in a humidified incubator with $5 \% \mathrm{CO}_{2}$ at $37{ }^{\circ} \mathrm{C}$ prior to stimulation. To characterize monocyte functionality, monocytes were exposed to lipopolysaccharide (LPS), which is the major component of the outer membrane of Gram-negative bacteria, in order to stimulate the immune response, and dexamethasone, a glucocorticoid known to have immunosuppressive properties, to test monocyte sensitivity to immunoregulation. Hence, after resting for $2 \mathrm{~h}$, monocytes were preincubated for $30 \mathrm{~min}$ with or without $100 \mathrm{nM}$ dexamethasone followed by stimulation for $24 \mathrm{~h}$ with $1 \mathrm{ng} / \mathrm{mL}$ LPS (Sigma-Aldrich, Saint Louis, MO, USA). LPS was dissolved in culture medium whereas a stock solution of dexamethasone $(1 \mathrm{mg} / \mathrm{mL})$ was prepared in ethanol and further diluted in culture medium. Ethanol had no effect on monocyte cultures at 
the concentration used in the experiment (final ratio of $1 / 25000$ ). Hence, sterile culture medium was used as negative control for all conditions. After $24 \mathrm{~h}$ of incubation, a supernatant was collected and cells were frozen. Supernatants were stored at $-20{ }^{\circ} \mathrm{C}$ until subsequent analysis.

\section{Cytokine quantification in cell culture supernatant}

For the analysis of monocyte functionality, inflammatory cytokine levels in culture supernatants were measured in basal conditions and after LPS or dexamethasone plus LPS exposure of purified monocytes using multiplex bead-based sandwich immunoassay with Luminex $\mathrm{xMAP}^{\circ}$ technology (Luminex, Austin, TX, USA). A commercially available 5plex panel including proinflammatory cytokines and chemokines (IL-1 $\beta$, IL-6, granulocyte-macrophage colonystimulating factor (GM-CSF), TNF- $\alpha$, and IL-8) (cat no. LHC0003, Invitrogen, Carlsbad, CA, USA) was used following the manufacturer's instructions. Briefly, supernatants $(25 \mu \mathrm{L})$ and standards were incubated with cytokinespecific capture antibodies coupled to fluorescent beads. Then, biotinylated detector antibodies were added, followed by streptavidin-phycoerythrin incubation. All standards and samples were analyzed in duplicate. Data were acquired using Luminex 200 system and analyzed with xPonent v3.1 software (Luminex, Austin, TX, USA). Cytokine levels were quantified using a five-parameter logistic regression curve derived from the reference cytokine concentration standards supplied by the manufacturer. The sensitivity of the assay allowed the detection of cytokine concentrations within the following ranges: IL- $1 \beta \quad 0.33-6800 \mathrm{pg} / \mathrm{mL}$, IL-6 $0.25-5000 \mathrm{pg} / \mathrm{mL}$, GM-CSF $0.77-15,400 \mathrm{pg} / \mathrm{mL}$, TNF- $\alpha$ $0.36-7300 \mathrm{pg} / \mathrm{mL}$, IL-8 $0.47-9500 \mathrm{pg} / \mathrm{mL}$. For experimental data above the range of the standard curve, the values were extrapolated, whereas samples below the lower limit of quantitation were assigned a value of one half of the minimum detection level for that cytokine as reported in previous studies [36, 37]. For the vast majority of the cytokines and assay conditions, at least $80 \%$ of the samples were within the limits of quantification. For some samples, it was not possible to determine cytokine concentration and so the following numbers of samples were included in the statistical analysis for each cytokine: IL-1 $\beta, 100$ OCD and 43 controls; IL-6, 79 OCD and 39 controls; GM-CSF, 100 OCD and 44 controls; TNF- $\alpha, 98$ OCD and 44 controls; and IL- 8,79 OCD and 40 controls.

In order to assess inflammatory activation of peripheral monocytes upon an immune challenge, percentages of cytokine production with respect to untreated sample were calculated for each cytokine level after treatment with LPS alone or LPS plus dexamethasone. Besides, to estimate monocyte sensitivity to dexamethasone, differences in cytokine concentrations between LPS-treated and LPS + dexamethasone-treated samples were computed and expressed as percentages with respect to LPS stimulation.

\section{Statistical analysis}

Data were analyzed using IBM SPSS statistics 20 (IBM Corp., Chicago, IL, USA). Normality was assessed using Kolmogorov-Smirnov and Shapiro-Wilk tests. The different immune parameters, including all percentages of monocyte subsets and the five cytokine levels in basal conditions and after LPS or LPS + dexamethasone treatment, were found to follow a non-normal distribution. Therefore, all variables were natural-log-transformed for subsequent analysis. In order to test differences in sociodemographic variables between patients and controls, Student's $t$ test for continuous variables and chi-square test for categorical variables were used. Pearson's test was also used to assess correlations. Univariate analyses were performed adjusting for gender and age to assess the difference in immune parameters between patients and controls. When a significant correlation was identified between cytokine levels and monocyte subsets, the corresponding monocyte subpopulation was introduced as a covariate in the univariate analysis. When the immune parameters were found to be associated with OCD, we also assessed their putative relationship with clinical variables such as disease severity, duration of disease, or psychopharmacological status. When appropriate, further pairwise comparison analyses were performed using Bonferroni's post hoc test.

All data are reported as means \pm standard error of the mean (SEM) of the original data, prior to log transformation. All tests were two-tailed and significance level was set at $p<0.05$.

\section{Results}

\section{Clinical and sociodemographic characteristics of the sample}

Demographic and clinical data of patients with OCD and the control group are shown in Table 1. The mean age was 14.78 years for OCD individuals and 16.29 years for healthy controls $(t=-3.608, p=0.0004)$. There were 52 boys $(51 \%)$ and 50 girls (49\%) in the case group and 14 boys $(29.8 \%)$ and $33(70.2 \%)$ girls among the controls $\left(\chi^{2}=5.86, p=0.016\right)$. The mean symptom severity score in OCD individuals, as assessed by the CY-BOCS scale was $25.97 \pm 0.64$; this high value reflected the pathological condition of patients. Some patients were also diagnosed with internalizing disorders (anxiety or depression disorders) $(N=46,45.1 \%)$ or with neurodevelopmental disorders (attention deficit hyperactivity disorder (ADHD) or tics) $(N=22,21.6 \%)$. However, the presence of these comorbidities in some OCD patients does not seem to have an effect on the frequency of 
Table 1 Demographic and clinical data of the study population

\begin{tabular}{|c|c|c|c|c|}
\hline & OCD patients $(N=102)$ & Controls $(N=47)$ & Statistic & $p$ value \\
\hline Male gender, $N(\%)$ & $52(51.0)$ & $14(29.8)$ & $x^{2}=5.86$ & $0.016^{\mathrm{a}}$ \\
\hline Age (mean \pm SEM) & $14.78 \pm 0.26$ & $16.29 \pm 0.26$ & $t=-3.608$ & $0.0004^{b}$ \\
\hline Age of onset (mean \pm SEM) & $12.95 \pm 0.28$ & - & - & - \\
\hline Duration of illness, months (mean \pm SEM) & $24.62 \pm 2.44$ & - & - & - \\
\hline CY-BOCS score (mean \pm SEM) & $25.97 \pm 0.64$ & - & - & - \\
\hline \multicolumn{5}{|l|}{ Comorbidities, N (\%) } \\
\hline Anxiety or mood disorders & $46(45.1)$ & - & - & - \\
\hline ADHD or tic disorder & $22(21.6)$ & - & - & - \\
\hline \multicolumn{5}{|l|}{ Treatment, N (\%) } \\
\hline Medicated & $82(80.4)$ & - & - & - \\
\hline Antidepressants & $59(72)$ & - & - & - \\
\hline Antipsychotics & $1(1.2)$ & - & - & - \\
\hline Antidepressants + antipsychotics & $22(26.8)$ & - & - & - \\
\hline Non-medicated & $20(19.6)$ & - & - & - \\
\hline
\end{tabular}

OCD obsessive compulsive disorder, $A D H D$ attention deficit hyperactivity disorder, $C Y$-BOCS Children's Yale-Brown Obsessive-Compulsive Scale, SEM standard error of the mean

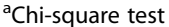

bstudent's $t$ test

monocyte subsets and cytokine levels, as no differences were found between the groups (Additional file 2: Table S1).

Eighty-two patients (80.4\%) received pharmacological treatment with an antidepressant alone $(N=59,72 \%)$, an antidepressant combined with antipsychotic drugs $(N=$ $22,26.8 \%)$, or an antipsychotic alone $(N=1,1.2 \%)$. The most prescribed antidepressant was fluoxetine (in $45.7 \%$ of patients receiving antidepressants), whereas the most used antipsychotic was risperidone (in $43.5 \%$ of patients taking antipsychotics). Ten patients were comedicated with benzodiazepines (12.2\%), four (4.9\%) with longacting methylphenidate, and one (1.2\%) with lithium carbonate.
Although a different number of samples was included in the analysis of monocyte subsets and the five proinflammatory cytokines, no differences were identified between these populations regarding any of the clinical and sociodemographic characteristics (data not shown).

\section{Enrichment of CD16+ monocytes in peripheral blood of OCD patients}

OCD patients presented higher percentages of total monocytes than healthy controls $(F=7.199, p=0.008)$ (Fig. 1a). Regarding different monocyte subsets, CD16+ monocytes were enriched in OCD patients compared to healthy controls $(F=18.188, p=0.00004)$ (Fig. 1b). This effect was
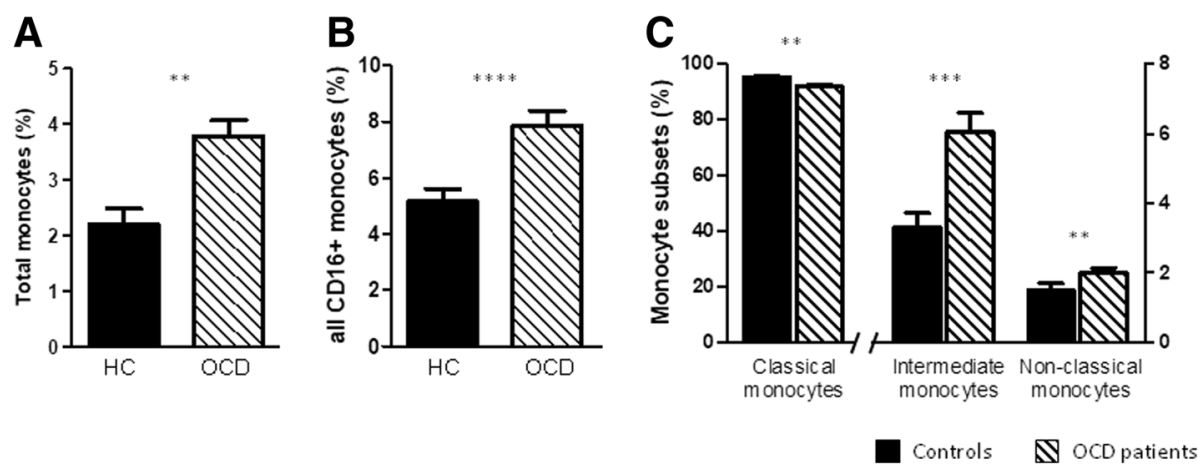

Fig. 1 Distribution of monocyte subsets in early-onset OCD patients $(N=91)$ and healthy controls $(H C, N=34)$. a Percentage of total monocytes in OCD patients and healthy controls. b Percentage of all CD16+ monocytes, including CD14 $4^{\text {high }} C D 16^{\text {low }}$ and $C D 14^{\text {low }} C D 16^{\text {high }}$ monocytes, in OCD patients and healthy controls. c Percentage of classical $\left(C D 14^{\text {high }} \mathrm{CD} 16-\right)$, intermediate $\left(C D 14^{\text {high }} \mathrm{CD} 16^{\text {low }}\right)$, and non-classical $\left(C D 14^{\text {low }} C D 16^{\text {high }}\right)$ monocytes in $O C D$ patients and healthy controls. Results are expressed as means \pm SEM of the original data, prior to log transformation. Statistical analysis was performed using univariate general linear model adjusted for age and gender with natural-log-transformed data. ${ }^{* *} p<0.01,{ }^{* * *} p<0.001,{ }^{* * *} p<0.0001$ 
largely driven by a twofold increase in intermediate monocytes in OCD patients $(F=15.013, p=0.0002)$ (Fig. 1c), although non-classical monocytes were also slightly higher in OCD individuals $(F=7.107, p=0.009)$. Concurrently, classical monocytes were slightly less frequent in patients than in controls $(F=10.188, p=0.002)$ (Fig. 1c).

\section{Higher production of inflammatory cytokines in OCD patients}

There was a high correlation between the levels of the five different cytokines measured in the study (Additional file 3: Table S2). In addition, cytokine levels were mainly significantly correlated with total monocytes but not with the different monocyte subsets (Additional file 4: Table S3).

No differences were identified in cytokine levels in basal conditions between patients and controls (Additional file 5: Table S4). Effects of monocyte stimulation with LPS or LPS + dexamethasone are shown in Fig. 2. After LPS treatment, OCD samples showed a significantly higher production of inflammatory cytokines (IL-1 $\beta, F=8.216 p=0.005$; IL-6, $F=8.570 p=0.004 ;$ GM-CSF, $F=4.433 p=0.049$; TNF- $\alpha, F=4.241 p=0.041$; IL-8, $F=6.330 p=0.013$ ) than healthy controls. As expected, cells pretreated with dexamethasone before LPS stimulation released lower quantities of inflammatory cytokines than those treated with LPS alone in both OCD patients and controls. However, cytokine levels after LPS + dexamethasone treatment were still higher than in basal conditions. Due to cytokine production after LPS stimulation, monocytes from OCD patients released higher quantities of the inflammatory cytokines than healthy controls after LPS + dexamethasone treatment. These differences were only significant for some of the mediators: IL-1 $\beta(F=5.528 ; p=0.020)$, IL-6 $(F=6.284 ; p=0.014), \quad \mathrm{GM}-\mathrm{CSF} \quad(F=2.205 ; p=0.140)$, TNF- $\alpha(F=1.576 ; p=0.211)$, and IL-8 $(F=10.326 ; p=$ $0.002)$. Nevertheless, monocyte sensitivity to dexamethasone expressed as a percentage of reduction in cytokine levels in LPS + dexamethasone-treated monocytes with respect to LPS-stimulated cells did not differ between patients and controls (Additional file 5: Table S4).

\section{Clinical characteristics of OCD patients and immune parameters}

We also addressed the possible effects of pharmacological treatment on monocyte distribution and cytokine production in cultured monocytes. We found a progressive increase in CD16+ and intermediate and nonclassical subsets from healthy subjects to OCD individuals receiving pharmacological treatment and finally to non-treated patients (CD16+ monocytes $F=10.372, p=$ 0.00007; intermediate monocytes $F=8.647, p=0.0003$; non-classical monocytes $F=3.561, p=0.032$ ) (Fig. 3a). In contrast, the direction of this association was reversed for classical monocytes, since the percentage of this subset was higher in healthy individuals than in treated patients and finally in unmedicated patients $(F=6.620$, $p=0.002$ ) (Fig. 3a). The same pattern was identified for cytokine levels after LPS stimulation: there was a

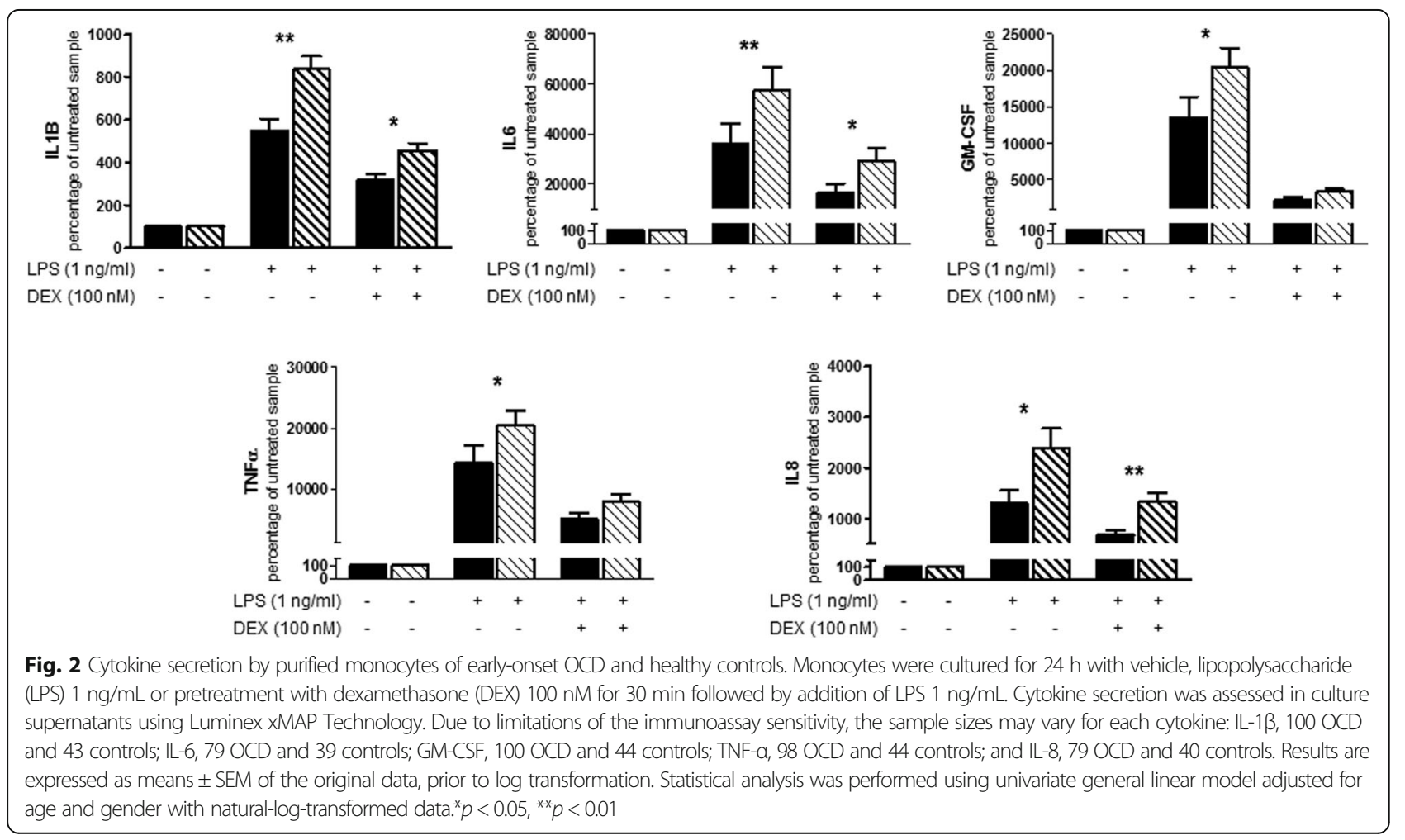



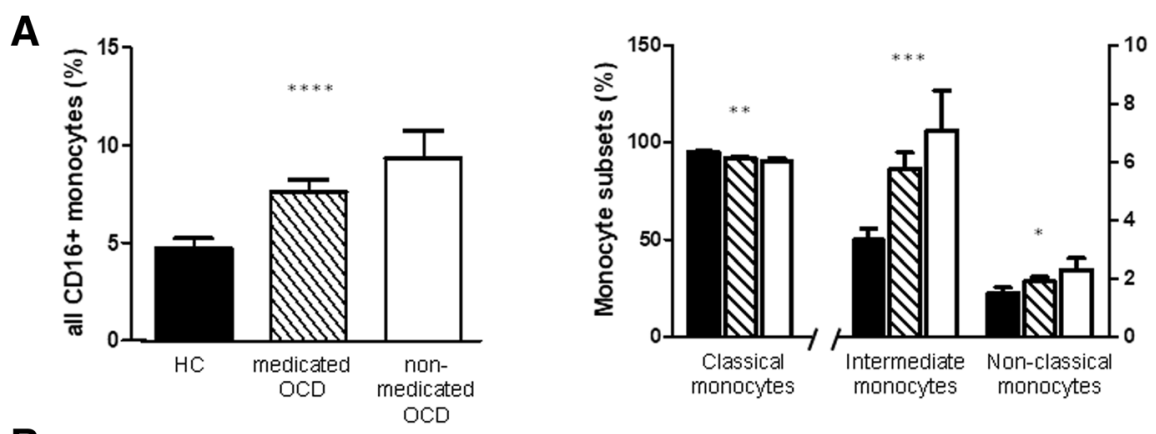

B

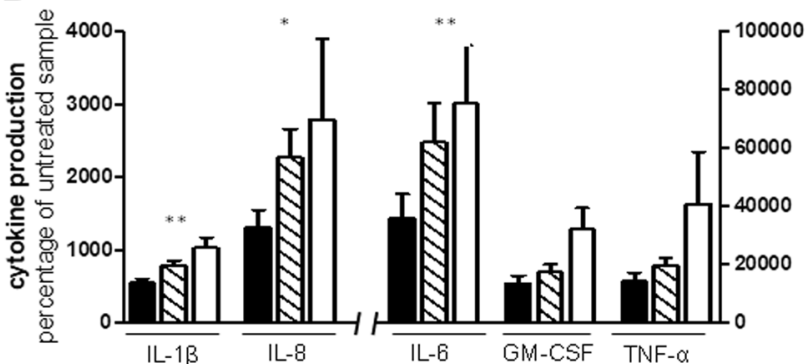

Controls

\$ Medicated OCD patients

$\square$ Non-medicated OCD patients

Fig. 3 Influence of medication on immune parameters in OCD. a Distribution of monocyte subsets (percentages of all CD16+, classical, intermediate, and non-classical monocytes) in peripheral blood of medicated $(N=71)$ and non-medicated patients $(N=20)$ with early-onset $O C D$ and healthy controls ( $\mathrm{HC}$, $N=34)$. b Cytokine secretion by purified monocytes of medicated and non-medicated patients with early-onset OCD and healthy controls after 24 h of stimulation with LPS $1 \mathrm{ng} / \mathrm{mL}$ (IL-1 43 controls, 80 medicated OCD, 20 non-medicated OCD; IL-8 40 controls, 62 medicated OCD, 17 non-medicated OCD; IL-6 39 controls, 66 medicated-OCD, 13 non-medicated OCD; GM-CSF 44 controls, 80 medicated OCD, 20 non-medicated OCD; TNF-a 44 controls, 79 medicated OCD, 19 non-medicated OCD). Results are expressed as means \pm SEM of the original data, prior to log transformation. Statistical analysis was performed using univariate general linear model adjusted for age and gender with natural-log-transformed data. ${ }^{*} p<0.05,{ }^{*} p<0.01$, ${ }^{* * *} p<0.001,{ }^{* * * *} p<0.0001$

progressive increase in cytokine concentrations from healthy subjects to OCD patients receiving pharmacological treatment and finally to patients without medication. This association was significant for most cytokines (IL-1 $\beta F=5.464, p=0.005 ;$ IL-6 $F=5.076, p=0.008$; GM-CSF $F=1.917, \quad p=0.151 ;$ TNF- $\alpha \quad F=2.102, \quad p=$ 0.126 ; and IL8 $F=4.526, p=0.013$, Fig. 3b). Post hoc comparisons revealed that healthy controls had lower levels of monocyte subsets and proinflammatory cytokines compared to both untreated and treated OCD patients $(p<0.05)$. However, no significant differences were found between OCD individuals receiving psychoactive medications and untreated patients.

In addition, we evaluated the putative relationship of monocyte subset frequency and cytokine production with severity of obsessive-compulsive symptoms as well as with duration of disease. None of the immune parameters were significantly correlated with these clinical variables (Additional file 6: Table S5).

\section{Discussion}

To our knowledge, this is the first study to evaluate the distribution of peripheral monocyte subsets and to analyze markers of monocyte activation and functionality in earlyonset OCD. Our results revealed a proinflammatory predisposition of monocytes from OCD patients based on the imbalance of monocyte subpopulations and their over-reactivity to immune stimulation with LPS. Moreover, OCD patients receiving psychoactive medications were found to have an intermediate inflammatory profile compared to untreated patients and controls.

OCD patients showed an increase in the percentage of total monocytes as well as in the CD16+ subset, especially in intermediate monocytes but also in non-classical monocytes, compared with healthy controls. Although there is no previous evidence of an association between altered monocyte frequencies and OCD, some authors have identified high levels of monocytes in related disorders like Tourette's syndrome (TS) [38] or autism spectrum disorder $[39,40]$. However, no differences in the monocyte subset distribution have been found [41].

Together with these alterations in monocyte subsets, we found that isolated peripheral blood monocytes from children with OCD behaved abnormally upon stimulation with LPS, displaying excessive IL-1 $\beta$, IL-6, GM-CSF, TNF- $\alpha$, and IL- 8 production compared to monocytes from healthy controls. Interestingly, we did not detect any abnormal activation of monocytes from OCD patients in basal conditions, but exposure to an immune challenge was required for the over-reactivity of these 
cells. Whether this over-reactivity occurs upon other types of immune stimulus such as psychological stress remains to be elucidated. These findings are at odds with results from previous studies with similar methodologies, which have reported either no differences or lower production of several cytokines after LPS stimulation in OCD individuals [42-45]. However, we should stress that in these studies, the assessments involved different types of samples including other immune cells and all of them were conducted in adult patients, in whom the pathological mechanism may be different $[4,5]$. In addition, cytokine production in children has been reported to be different from that in adults $[46,47]$. Apart from the studies mentioned above, most authors have investigated cytokine levels in serum or cerebrospinal fluid in OCD [16-18, 47, 48] but the results were inconsistent due to methodological and sample differences such as age, age at onset, disease duration, and pharmacological treatment. Interestingly, in our population, monocytes from pediatric OCD patients were able to respond to glucocorticoid exposure in the same way as those from healthy individuals. However, due to the over-reactivity of monocytes from OCD patients after LPS stimulation, cytokine levels remained higher in patients than in controls when treated with dexamethasone. The significant correlation identified between cytokine levels and the percentage of total monocytes indicates that those individuals with higher production of inflammatory cytokines, both in basal conditions and in response to stimulus, were also those with the higher levels of monocytes and thus with a greater global inflammatory state. However, as all the samples were cultured using the same amount of monocytes, we can rule out that the amount of cytokines released was due to the number of monocytes. In addition, as cytokine levels were mainly not correlated with the different monocyte subsets, the higher production of cytokines must be due to a higher activation of monocytes rather than to monocyte subset distribution.

This study also found that OCD patients receiving pharmacological treatment seem to present an intermediate inflammatory profile (including monocyte subset distribution and cytokine production upon stimulation with LPS) that is lower than non-medicated OCD individuals and higher than healthy controls. These results are in accordance with previous evidence suggesting that treatment with antidepressant medications may have anti-inflammatory properties $[49,50]$. However, post hoc analysis was not able to detect significant differences between treated and untreated patients, maybe due to the small size of the non-medicated group. If the trend identified here is confirmed in subsequent studies, the combined treatment with antidepressants and anti-inflammatory drugs like glucocorticoids may be a promising therapy for improving obsessive-compulsive symptoms, as observed in patients with major depressive disorder or schizophrenia [51, 52]. In addition, other immune-modulating medications, such as anticytokine agents, may be useful as adjunct therapy in OCD individuals, as observed in a patient with tumor necrosis factor receptor-associated autoinflammatory syndrome (TRAPS), in which psychiatric symptoms, including tics and OCD, showed a remarkable improvement after treatment with the IL-1 blocking agent anakinra [53].

Monocytes, which were found to be increased in OCD patients in the present study, are the main cells implicated in the first immune response upon infection. Among the different monocyte subsets, CD16+ and specifically the intermediate monocytes, which were increased in our OCD population, are considered the most inflammatory subpopulation due to their expression of inflammatory cytokines and other activation markers and due to their expansion in infectious and inflammatory diseases [29-31]. Circulating monocytes can be recruited to the central nervous system (CNS) upon exposure to psychosocial stress [25] or when chronic or intense injury occurs in the brain, where they contribute to the inflammatory response with their phagocytic activity and the release of immune mediators such as cytokines $[28,54]$. Additionally, peripherally produced cytokines secreted by monocytes, such as the IL-1 $\beta$, IL-6, GM-CSF, TNF- $\alpha$, and IL- 8 evaluated in this study, not only act peripherally but also enter the CNS where they increase neuroinflammatory responses and affect neurotransmitter availability [55, 56], brain function, and neurodevelopment [57]. These effects occur mainly in the basal ganglia and dorsal anterior cingulate cortex [58], which have been implicated in the pathophysiology of OCD [59]. Hence, monocyte activation upon immune challenges and other potential stressors in genetically susceptible individuals may be involved in the onset, progression, and exacerbation of obsessivecompulsive symptoms, as proposed for other psychiatric diseases such as schizophrenia [23, 60], depression [58] or anxiety [21]. This peripheral activation of monocytes may also suggest microglial activation, since they are the innate immune cells of the CNS. Although microglia originate from myeloid precursors deriving from the yolk sac during embryonic development, with minimal contribution of bone marrow-derived progenitors in adulthood [54, 61], monocytes and microglial cells may exhibit similar responses to systemic stimuli [54, 62]. Activated microglia with a proinflammatory phenotype are unable to remove debris and promote regeneration of the inflamed tissue leading to a failure in immune resolution and neuroprotection [28]. In addition, microglial abnormalities may lead to alterations of synaptic pruning as well as higher release of microglial-derived 
glutamate, which in turn might have neurotoxic effects on dendrites and synapses [63]. Indeed, it has been suggested that microglial dysregulation may have a role in the pathophysiology of OCD and related diseases like TS or autism. Postmortem studies evaluating gene expression in basal ganglia from TS subjects identified an increase in the expression of monocyte chemotactic factor-1 (MCP-1) [64] and an upregulation of several microglia-related genes [65], pointing to microglia proliferation and activation. In addition, animal models such as Hoxb8 knockout mice or L-histidine decarboxylase ( $H d c)$ knockout mice, exhibiting repetitive behaviors, mostly grooming, have also suggested the participation of microglia in the development of OCD and TS [63, 66, 67].

Taken together, our results indicate an enhanced proinflammatory state in monocytes in early-onset OCD characterized by alterations in monocyte subset distribution as well as higher production of inflammatory cytokines after monocyte stimulation. It should be borne in mind that our sample comprised OCD patients with a variety of comorbidities and that most patients were receiving pharmacological treatment. However, the higher activation state of monocytes from OCD patients identified here was not due to the presence of these conditions. We should also stress the homogeneity of our OCD sample in terms of age, age at onset, or duration of disease, as all participants were children and adolescents; this avoids the possible influence of these confounding factors and allows the study of the specific etiopathogenic mechanism underlying childhood-onset OCD, which may differ from the pathophysiology of the adult disorder [5]. In addition, a larger size of the control sample, at least similar to that of the OCD group, would be preferred. However, due to the frequent difficulties found in the recruitment of healthy children and adolescents, we were not able to include a higher number of controls. Moreover, despite the imbalance in gender between OCD patients and healthy controls and the age difference between the groups, the results of the univariate analysis adjusted for these two factors revealed that the association between OCD and immune parameters that was identified here was independent of their potential confounding effect.

As we used a primary model of isolated monocytes, the enhanced proinflammatory state in OCD patients shown in this study was not influenced by the immune response mediated by other immune cells. This means that we can specifically determine the potential contribution of monocyte lineage cells to the inflammatory response in early-onset OCD. Nevertheless, although the over-activation of monocytes found in the present study was demonstrated by a number of parameters, only peripheral activation markers were measured. Hence, even though peripheral monocytes and circulating cytokines are able to enter the brain [55] and although these peripheral changes may also reflect microglial activation [23], analysis of more inflammatory markers, including central immune changes, is needed in order to understand the complex inflammatory profile underlying OCD. In addition to these limitations, it should also be borne in mind that no adjustments for multiple comparisons were applied due to the exploratory nature of the study and the high correlation of the monocyte-derived products. Hence, the results should be interpreted with caution.

\section{Conclusions}

In conclusion, the results of the present study point to a proinflammatory state of monocytes from children and adolescents with OCD. The enhanced activation of the innate immune system may be triggered by exposure to immunomodulatory stimuli like stress or infections leading to an increased production of cytokines that mediate the inflammatory process. Further studies are necessary to elucidate the role of innate immunity in the complex pathophysiology of OCD and to understand the interplay between neural and immune cells throughout the different stages of development.

\section{Additional files}

Additional file 1: Figure S1. Gating strategy for identification of monocyte subpopulations. (DOCX $324 \mathrm{~kb}$ )

Additional file 2: Table S1. Analysis of the percentages of total monocytes, monocyte subpopulations, and cytokine levels after LPS stimulation of purified monocytes in early-onset OCD diagnosed with different comorbidities. (DOCX $22 \mathrm{~kb}$ )

Additional file 3: Table S2. Correlations between the five proinflammatory cytokines measured in the study in basal conditions and after LPS or LPS-dexamethasone stimulation. (DOCX $21 \mathrm{~kb}$ )

Additional file 4: Table S3. Correlations between the monocyte subsets and the levels of the five proinflammatory cytokines measured in the study in basal conditions and after LPS or LPS-dexamethasone stimulation. (DOCX 23 kb)

Additional file 5: Table S4. Cytokine secretion by purified monocytes of early-onset OCD and healthy controls. (DOCX $24 \mathrm{~kb}$ )

Additional file 6: Table S5. Correlations of the duration of disease (expressed in months) and symptom severity (assessed by CY-BOCS score), with the percentage of total monocytes, monocyte subpopulations, and cytokine levels after LPS stimulation of monocytes in early-onset OCD. (DOCX $13 \mathrm{~kb}$ )

\section{Abbreviations}

ADHD: Attention deficit hyperactivity disorder; CNS: Central nervous system; CY-BOCS: Children's Yale-Brown obsessive-compulsive scale; DSMIV: Diagnostic and Statistical Manual of Mental Disorders; FBS: Fetal bovine serum; GM-CSF: Granulocyte-macrophage colony-stimulating factor; II1: Interleukin 1; IL-1 3 : Interleukin 1 beta; IL-6: Interleukin 6; IL-8: Interleukin 8; K-SADS-PL: Schedule for affective disorders and schizophrenia for school-age children-present and lifetime version; LPS: Lipopolysaccharide; OCD: Obsessive-compulsive disorder; PANDAS: Pediatric Autoimmune Neuropsychiatric Disorders Associated with Streptococcal Infections; PBMC: Peripheral blood mononuclear cell; PBS: Phosphate buffer saline: SC: Sydenham's chorea; TNF-a: Tumor necrosis factor alpha; TRAPS: Tumor necrosis factor receptor-associated autoinflammatory syndrome;

TS: Tourette's syndrome 


\section{Acknowledgements}

The authors thank the Language Advisory Service at the University of Barcelona, Spain, for manuscript revision. The authors also thank all subjects and their families for the time and effort spent on this study as well as Ana Meseguer for sample collection assistance.

\section{Funding}

This study was supported by the Alicia Koplowitz Foundation; the Ministerio de Economía y Competitividad, Instituto de Salud Carlos III, Fondo de Investigación Sanitaria (FIS) (PI13/01767); Spanish Ministry of Education, Culture and Sport (grant FPU14/06834 for D.B.), University of Barcelona (grant APIF2015_24782 for N.R.); and Hospital Clínic de Barcelona (grant Josep Font for EAGN). Support was also given by the "Agència de Gestió d'Ajuts Universitaris i Recerca" (AGAUR) of the "Generalitat de Catalunya" to the "Child Psychiatry and Psychology Group" (2014 SGR 489) and to the "Clinical Pharmacology and Pharmacogenetics Group" (2014 SGR 436).

\section{Availability of data and materials}

The datasets used and/or analyzed during the current study are available from the corresponding author on reasonable request.

\section{Authors' contributions}

NR and AM participated in designing and carrying out of the experimental procedures, performing the statistical analyses and the interpretation of results, and writing the first draft of the manuscript. EAGN, DB, TT, and SGC participated in carrying out the experimental procedures and helped in the drafting of the manuscript. PG, SM, and CS participated in the design of the study, the interpretation of the results, and the drafting of the manuscript. LZ and AM participated in the coordination, conceiving, and design of the study and helped in the drafting of the manuscript. LZ and AM provided clinical samples, helped in the drafting of the manuscript, and acquired funding for the research. All authors read and approved the final manuscript.

\section{Ethics approval and consent to participate}

All procedures were approved by the hospital's ethics committee. Written informed consent was obtained from all parents and verbal informed consent was given by all subjects following an explanation of the procedures involved.

\section{Consent for publication}

Not applicable

\section{Competing interests}

The authors declare that they have no competing interests.

\section{Publisher's Note}

Springer Nature remains neutral with regard to jurisdictional claims in published maps and institutional affiliations.

\section{Author details \\ 'Department of Basic Clinical Practice, University of Barcelona, Barcelona, Spain. ${ }^{2}$ Department of Child and Adolescent Psychiatry and Psychology, Institute of Neurosciences, Hospital Clinic de Barcelona, Barcelona, Spain. ${ }^{3}$ Immunology Service, Hospital Clinic de Barcelona, Barcelona, Spain. ${ }^{4}$ Department of Medicine, University of Barcelona, Barcelona, Spain. ${ }^{5}$ Department of Biomedicine, University of Barcelona, Barcelona, Spain. ${ }^{6}$ Institut d'Investigacions Biomèdiques August Pi i Sunyer (IDIBAPS), Barcelona, Spain. ${ }^{7}$ Centro de Investigación Biomédica en Red de Salud Mental (CIBERSAM), Madrid, Spain.}

Received: 4 October 2017 Accepted: 13 December 2017 Published online: 28 December 2017

\section{References}

1. American Psychiatric Association. Diagnostic and statistical manual of mental disorders. 5th ed. Washington: American Psychiatric Press; 2013.

2. Ruscio A, Stein D, Chiu W, Kessler R. The epidemiology of obsessivecompulsive disorder in the National Comorbidity Survey Replication. Mol Psychiatry. 2010;15:53-63.
3. Stewart SE, Geller DA, Jenike M, Pauls D, Shaw D, Mullin B, et al. Long-term outcome of pediatric obsessive-compulsive disorder: a meta-analysis and qualitative review of the literature. Acta Psychiatr Scand. 2004;110:4-13.

4. Dell'Osso B, Benatti B, Hollander E, Fineberg N, Stein DJ, Lochner C, et al. Childhood, adolescent and adult age at onset and related clinical correlates in obsessive-compulsive disorder: a report from the International College of Obsessive-Compulsive Spectrum Disorders (ICOCS). Int J Psychiatry Clin Pract. 2016;20:210-7.

5. Taylor S. Early versus late onset obsessive-compulsive disorder: evidence for distinct subtypes. Clin Psychol Rev. 2011;31:1083-100.

6. da Rocha FF, Correa H, Teixeira AL. Obsessive-compulsive disorder and immunology: a review. Prog Neuro-Psychopharmacology Biol Psychiatry 2008:32:1139-1146.

7. Teixeira AL, Rodrigues DH, Marques AH, Miguel EC, Fontenelle LF. Searching for the immune basis of obsessive-compulsive disorder. Neuroimmunomodulation. 2014;21:152-8.

8. Morer A, Viñas O, Lázaro L, Calvo R, Andrés S, Bosch J, et al. Subtyping obsessive-compulsive disorder: clinical and immunological findings in child and adult onset. J Psychiatr Res. 2006;40:207-13.

9. Swedo S, Rapoport J, Cheslow D, Leonard H, Ayoub E, Hosier D, et al. Highprevalence of obsessive-compulsive symptoms in patients with Sydenham's chorea. Am J Psychiatry. 1989;146:246-9.

10. Swedo SE, Leonard HL, Garvey M, Mittleman B, Allen AJ, Perlmutter S, et al. Pediatric autoimmune neuropsychiatric disorders associated with streptococcal infections: clinical description of the first 50 cases. Am J Psychiatry. 1998;155:264-71.

11. Macerollo A, Martino D. Pediatric autoimmune neuropsychiatric disorders associated with streptococcal infections (PANDAS): an evolving concept. Tremor Other Hyperkinet Mov (N Y). 2013;3 http://tremorjournal.org/article/view/167

12. Mercadante MT, Busatto GF, Lombroso PJ, Prado L, Rosario-Campos MC, do Valle $R$, et al. The psychiatric symptoms of rheumatic fever. Am J Psychiatry. 2000;157:2036-8.

13. Asbahr FR, Garvey MA, Snider LA, Zanetta DM, Elkis H, Swedo SE. Obsessivecompulsive symptoms among patients with Sydenham chorea. Biol Psychiatry. 2005;57:1073-6.

14. Allen AJ, Leonard HL, Swedo SE. Case study: a new infection-triggered, autoimmune subtype of pediatric OCD and Tourette's syndrome. J Am Acad Child Adolesc Psychiatry. 1995;34:307-11.

15. Swedo SE, Leckman JF, Rose NR. From research subgroup to clinical syndrome: modifying the PANDAS criteria to describe PANS (pediatric acute-onset neuropsychiatric syndrome). Pediatr Ther. 2012;2:113.

16. Gray SM, Bloch MH. Systematic review of proinflammatory cytokines in obsessive- compulsive disorder. Curr Psychiatry Rep. 2012;14:220-8.

17. Şimşek Ş, Yüksel T, Çim A, Kaya S. Serum cytokine profiles of children with obsessive-compulsive disorder shows the evidence of autoimmunity. Int J Neuropsychopharmacol. 2016;19:1-6.

18. Rao NP, Venkatasubramanian G, Ravi V, Kalmady S, Cherian A, YC JR. Plasma cytokine abnormalities in drug-naive, comorbidity-free obsessive-compulsive disorder. Psychiatry Res. 2015;229:949-52.

19. Dale RC, Heyman I, Giovannoni G, Church AWJ. Incidence of anti-brain antibodies in children with obsessive-compulsive disorder. Br J Psychiatry. 2005;187:314-9.

20. Morer A, Lázaro L, Sabater L, Massana J, Castro J, Graus F. Antineuronal antibodies in a group of children with obsessive-compulsive disorder and Tourette syndrome. J Psychiatr Res. 2008;42:64-8.

21. Furtado M, Katzman MA. Neuroinflammatory pathways in anxiety, posttraumatic stress, and obsessive compulsive disorders. Psychiatry Res. 2015;229:37-48

22. Şimşek Ş, Gençoğlan S, Yüksel T, Kaplan I, Alaca R. Cortisol and brain-derived neurotrophic factor levels prior to treatment in children with obsessivecompulsive disorder. J Clin Psychiatry. 2016;77:e855-9.

23. Bergink V, Gibney SM, Drexhage HA. Autoimmunity, inflammation, and psychosis: a search for peripheral markers. Biol Psychiatry. 2014;75:324-31.

24. Beumer W, Gibney SM, Drexhage RC, Pont-Lezica L, Doorduin J, Klein HC, et al. The immune theory of psychiatric diseases: a key role for activated microglia and circulating monocytes. J Leukoc Biol. 2012;92:959-75.

25. Wohleb ES, McKim DB, Sheridan JF, Godbout JP. Monocyte trafficking to the brain with stress and inflammation: a novel axis of immune-to-brain communication that influences mood and behavior. Front Neurosci. 2015;8:447.

26. Frick LR, Williams $K$, Pittenger $C$. Microglial dysregulation in psychiatric disease. Clin Dev Immunol. 2013;2013:608654. 
27. Auffray C, Sieweke MH, Geissmann F. Blood monocytes: development, heterogeneity, and relationship with dendritic cells. Annu Rev Immunol. 2009;27:669-92.

28. London A, Cohen M, Schwartz M. Microglia and monocyte-derived macrophages: functionally distinct populations that act in concert in CNS plasticity and repair. Front Cell Neurosci. 2013;7:34.

29. Wong KL, Yeap WH, Tai JJY, Ong SM, Dang TM, Wong SC. The three human monocyte subsets: implications for health and disease. Immunol Res. 2012;53:41-57.

30. Ziegler-Heitbrock L. The CD14+CD16+ blood monocytes: their role in infection and inflammation. J Leukoc Biol. 2007;81:584-92.

31. Ziegler-Heitbrock $L$, Hofer TPJ. Toward a refined definition of monocyte subsets. Front Immunol. 2013;4:23.

32. American Psychiatric Association. Diagnostic and statistical manual of mental disorders. 4th ed. Washington: American Psychiatric Press; 1994.

33. Ulloa RE, Ortiz S, Higuera F, Nogales I, Fresán A, Apiquian R, et al. Interrater reliability of the Spanish version of schedule for affective disorders and schizophrenia for school-age children-present and lifetime version (K-SADSPL). Actas Esp Psiquiatr. 2006;34:36-40.

34. Kaufman J, Birmaher B, Brent D, Rao U, Flynn C, Moreci P, et al. Schedule for affective disorders and schizophrenia for school-age children-present and lifetime version (K-SADS-PL): initial reliability and validity data. J Am Acad Child Adolesc Psychiatry. 1997;36:980-8

35. Scahill L, Riddle MA, McSwiggin-Hardin M, Ort SI, King RA, Goodman WK, et al. Children's Yale-Brown obsessive compulsive scale: reliability and validity. J Am Acad Child Adolesc Psychiatry. 1997;36:844-52.

36. Ashwood P, Krakowiak P, Hertz-Picciotto I, Hansen R, Pessah I, Van de Water J. Elevated plasma cytokines in autism spectrum disorders provide evidence of immune dysfunction and are associated with impaired behavioral outcome. Brain Behav Immun. 2011;25:40-5

37. Manzardo AM, Henkhaus R, Dhillon S, Butler MG. Plasma cytokine levels in children with autistic disorder and unrelated siblings. Int J Dev Neurosci. 2012;30:121-7.

38. Matz J, Krause DL, Dehning S, Riedel M, Gruber R, Schwarz MJ, et al. Altered monocyte activation markers in Tourette's syndrome: a case-control study. BMC Psychiatry. 2012;12:29.

39. Denney DR, Frei BW, Gaffney GR. Lymphocyte subsets and interleukin-2 receptors in autistic children. J Autism Dev Disord. 1996;26:87-97.

40. Sweeten TL, Posey DJ, McDougle CJ. High blood monocyte counts and neopterin levels in children with autistic disorder. Am J Psychiatry. 2003;160:1691-3.

41. Enstrom AM, Onore CE, Van De Water JA. Differential monocyte responses to TLR ligands in children with autism spectrum disorders. Brain Behav Immun. 2010;24:64-71

42. Fluitman S, Denys D, Vulink N, Schutters S, Heijnen C, Westenberg H. Lipopolysaccharide-induced cytokine production in obsessive-compulsive disorder and generalized social anxiety disorder. Psychiatry Res. 2010;178:313-6.

43. Fluitman SB, Denys DA, Heijnen CJ, Westenberg HG. Disgust affects TNF-a, IL-6 and noradrenalin levels in patients with obsessive-compulsive disorder. Psychoneuroendocrinology. 2010;35:906-11.

44. Denys D, Fluitman S, Kavelaars A, Heijnen C, Westenberg H. Decreased TNF$a$ and NK activity in obsessive-compulsive disorder

Psychoneuroendocrinology. 2004;29:945-52.

45. Denys D, Fluitman S, Kavelaars A, Heijnen C, Westenberg HGM. Effects of paroxetine and venlafaxine on immune parameters in patients with obsessive compulsive disorder. Psychoneuroendocrinology. 2006;31:355-60.

46. Lilic D, Cant AJ, Abinun M, Calvert JE, Spickett GP. Cytokine production differs in children and adults. Pediatr Res. 1997:42:237-40.

47. Konuk N, Tekin IO, Ozturk U, Atik L, Atasoy N, Bektas S, et al. Plasma levels of tumor necrosis factor-alpha and interleukin-6 in obsessive compulsive disorder. Mediat Inflamm. 2007;2007:65704.

48. Gabbay V, Coffey BJ, Guttman LE, Gottlieb L, Katz Y, Babb JS, et al. A cytokine study in children and adolescents with Tourette's disorder. Prog Neuro-Psychopharmacology Biol Psychiatry 2009;33:967-971.

49. Maes M. The immunoregulatory effects of antidepressants. Hum Psychopharmacol. 2001;16:95-103.

50. Caiaffo V, Oliveira BDR, de Sá FB, Evêncio Neto J. Anti-inflammatory, antiapoptotic, and antioxidant activity of fluoxetine. Pharmacol Res Perspect. 2016:4:e00231

51. Na KS, Lee KJ, Lee JS, Cho YS, Jung HY. Efficacy of adjunctive celecoxib treatment for patients with major depressive disorder: a meta-analysis. Prog Neuro-Psychopharmacology Biol Psychiatry 2014;48:79-85.
52. Keller WR, Kum LM, Wehring HJ, Koola MM, Buchanan RW, Kelly DL. A review of anti-inflammatory agents for symptoms of schizophrenia. J Psychopharmacol. 2013;27:337-42.

53. Garcia-Delgar B, Morer A, Luber MJ, Coffey BJ. Obsessive-compulsive disorder, tics, and autoinflammatory diseases: beyond PANDAS. J Child Adolesc Psychopharmacol. 2016;26:847-50.

54. Prinz M, Priller J. Microglia and brain macrophages in the molecular age: from origin to neuropsychiatric disease. Nat Rev Neurosci. 2014;15:300-12.

55. Felger JC, Lotrich FE. Inflammatory cytokines in depression: neurobiological mechanisms and therapeutic implications. Neuroscience. 2013;246:199-229.

56. Dunn A. Effects of cytokines and infections on brain neurochemistry. Clin Neurosci Res. 2006;6:52-68

57. Doherty $\mathrm{GH}$. Developmental switch in the effects of TNFa on ventral midbrain dopaminergic neurons. Neurosci Res. 2007;57:296-305.

58. Miller $\mathrm{AH}$, Maletic $\mathrm{V}$, Raison $\mathrm{CL}$. Inflammation and its discontents: the role of cytokines in the pathophysiology of major depression. Biol Psychiatry. 2009;65:732-41.

59. Pauls DL, Abramovitch A, Rauch SL, Geller DA. Obsessive-compulsive disorder: an integrative genetic and neurobiological perspective. Nat Rev Neurosci. 2014;15:410-24.

60. Benros ME, Mortensen PB, Eaton WW. Autoimmune diseases and infections as risk factors for schizophrenia. Ann N Y Acad Sci. 2012;1262:56-66.

61. Tay $T L$, Hagemeyer N, Prinz M. The force awakens: insights into the origin and formation of microglia. Curr Opin Neurobiol. 2016:39:30-7.

62. Takahashi Y, Yu Z, Sakai M, Tomita H. Linking activation of microglia and peripheral monocytic cells to the pathophysiology of psychiatric disorders. Front Cell Neurosci. 2016;10:144.

63. Frick L, Pittenger C. Microglial dysregulation in OCD, Tourette syndrome, and PANDAS. J Immunol Res. 2016;2016:8606057.

64. Morer A, Chae W, Henegariu O, Bothwell ALM, Leckman JF, Kawikova I. Elevated expression of MCP-1, IL-2 and PTPR-N in basal ganglia of Tourette syndrome cases. Brain Behav Immun. 2010;24:1069-73.

65. Lennington JB, Coppola G, Kataoka-Sasaki Y, Fernandez TV, Palejev D, Li Y, et al. Transcriptome analysis of the human striatum in Tourette syndrome. Biol Psychiatry. 2016;79:372-82.

66. Chen S, Tvrdik P, Peden E, Cho S, Wu S, Spangrude G, et al. Hematopoietic origin of pathological grooming in Hoxb8 mutant mice. Cell. 2010;141:775-85.

67. Greer JM, Capecchi MR. Hoxb8 is required for normal grooming behavior in mice. Neuron. 2002:33:23-34.

\section{Submit your next manuscript to BioMed Central and we will help you at every step:}

- We accept pre-submission inquiries

- Our selector tool helps you to find the most relevant journal

- We provide round the clock customer support

- Convenient online submission

- Thorough peer review

- Inclusion in PubMed and all major indexing services

- Maximum visibility for your research

Submit your manuscript at www.biomedcentral.com/submit

) Biomed Central 